\title{
THE DEVELOPMENT OF MONITORING AND CONTROL SYSTEM OF THE LOW PVIT SOLAR SYSTEM
}

\author{
Alena Okhorzina ${ }^{1,2, *}$, Alexander Bikbulatov ${ }^{1}$, Alexey Yurchenko ${ }^{1,2}$, Norbert Bernhard ${ }^{3}$, \\ and Oksana Aldoshina ${ }^{1}$ \\ ${ }^{1}$ National Research Tomsk Polytechnic University, 634050, Tomsk, Russia \\ ${ }^{2}$ National Research Tomsk State University, 634050, Tomsk, Russia \\ ${ }^{3}$ Anhalt University of Applied Sciences, 06366, Köthen, Germany
}

\begin{abstract}
The article presents an autonomous PV/T solar installation. Installing converts solar energy into electricity and heat. The description of its components and elements that enhance its effectiveness shows. The description of the control program and control of the installation is given. The control system provides for tracking the sun and cooling the photovoltaic module.
\end{abstract}

\section{Introduction}

One of the decisive factors, preventing solar energy from being widely used, are the costs of solar energy systems. Cost reduction of photoelectric systems, generating electrical energy, is possible by reducing the costs of photoelectric modules or increasing the efficiency of such modules and the solar stations, they are built in [1].

One method of increasing the energy production is to use solar concentrators. Systems with built-in concentrators use mirrors and lenses to focus the incoming sunlight. The construction of photoelectric concentrators comes with several manufacturing problems: it has to reflect light to a given direction, be adequate to mass production, be able to keep high concentrations, be insensitive to manufacturing inaccuracies and installation, and be able to ensure consistent illumination of the photovoltaic modules surface. Concentrator based solar systems turn out to be highly efficient, when steadily cooled in form of heat dissipation.

Heretofore there was no necessity for a widespread use of alternative energy sources in Russia, since Russia occupies a huge amount of traditional energy sources, such as oil, coal, gas, water and atomic energy, and it has a fitting infrastructure. As a result of the financial crisis, though, the delivery of combustible materials to remote and elusive places suffers.

Therefore, the authors see great prospects for alternative energy in providing energy resources of small settlements and private houses in remote settlements and holiday villages (for example, a village in the forest, forest wardens houses, national parks and reserves).

* Corresponding author: ohra.avit@gmail.com 


\section{Installation description}

The installation is a hybrid system (Figure 1), which converts solar energy into electricity and heat. The reflected sunlight incident on the photovoltaic module is converted into electrical energy. Heat production is due to the cooling of the PV.

To increase the energy production is added in the low reflective concentrator. The concentrator is a reflecting surface that consists of three segments. The concentrator provides increased density of the incoming solar energy flow in $2 \ldots 3$ times, thereby increasing the efficiency and the production of electricity. Also because of the increase in incoming solar flux on the surface of the PV module is heated; which leads to loss of efficiency and, when heated to a temperature above $130^{\circ} \mathrm{C}$, delamination or deterioration of elements of ohmic contacts photocells [2, 3].

It is therefore necessary to add cooling system reliability. It is possible to use different radiators or liquid cooling. Heat transferred by the cooling liquid, may be used for further purposes (ex for water heating). Thus, a combined system is obtained, which can generate both electricity and heat.

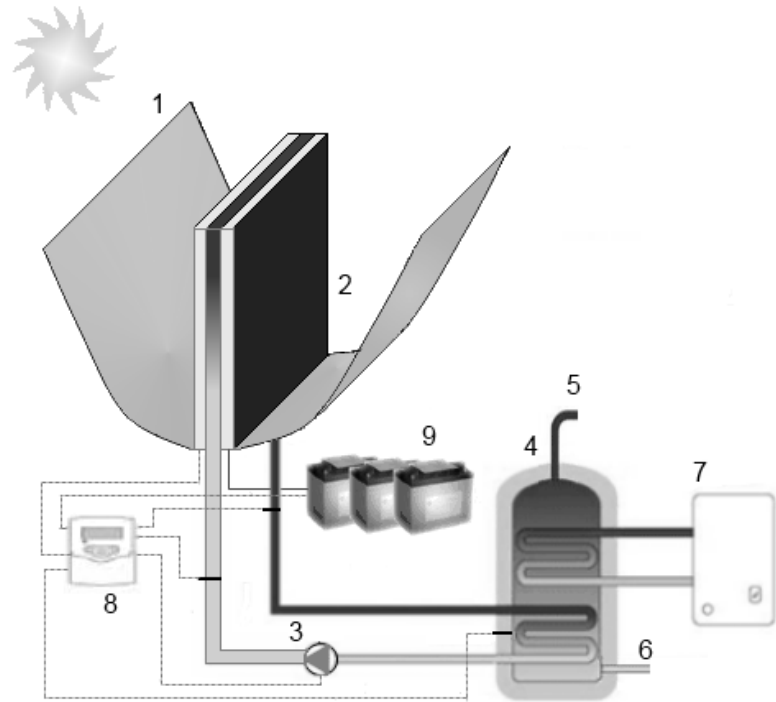

Figure 1. PV concentrator system with a heat sink: 1 - Concentrator, 2 - PV module, 3 - Pump, 4 - Twin coal, 5 - Output, 6 - Input, 7 - Boiler, 8 - Control system, 9 - Accumulator.

The application concentration and tracking the sun can increase the efficiency of the solar energy system, resulting in a more uniform production of electricity from sunrise to sunset.

\section{Monitoring and control system}

To ensure the end-consumer a simply and steadily working solar station, it is necessary to have a monitoring and control system. The given system ensures flawless functioning of the solar station and requires intervention only in the case of an error.

Overall control of the system will be based on a single-board computer Beaglebon. Beaglebon comprises a processor Sitara AM3359AZCZ100 series with clock speed of 1 $\mathrm{GHz}$, also processing core Cortex-A8 and SGX530 graphics accelerator. The board uses 512MB DDR3L RAM, Flash memory eMMC volume of 2 GB [4]. Data features are more than enough for a wide range of monitoring and control tasks. 
At the start of the system several actions are carried out, such as the initialization of the single-board computer Beaglebone and the start of the monitoring program.

The functional principles of the program algorithm are shown in Figure 2.

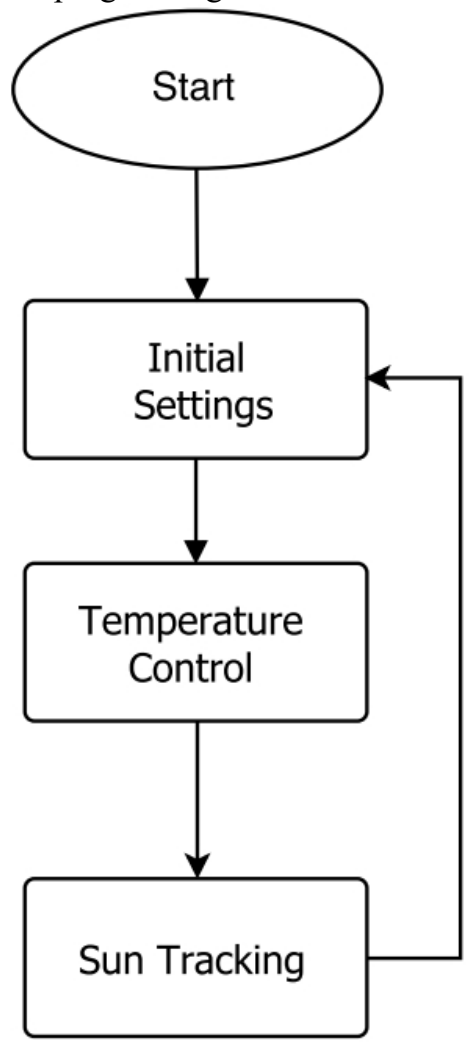

Figure 2. The program algorithm.

The given program monitors and controls the temperature of the PV by using liquid cooling. The computer controls the pump, which facilitates different speeds of circulation of the liquid. The higher the temperature of the PV, the higher is the speed of circulation of the liquid. Another job of the monitoring and control system is to orientate the whole station towards the sun to increase the received energy flux coming from the sun. This leads to a higher and more homogeneous energy input during the day. The controlling algorithm and the sensor of the orientation system are used for the orientation of the authors' earlier tracking system [5]

In the following the algorithm of the temperature-controlling subprogram will be explained in detail. The algorithm is shown in Figure 3. 


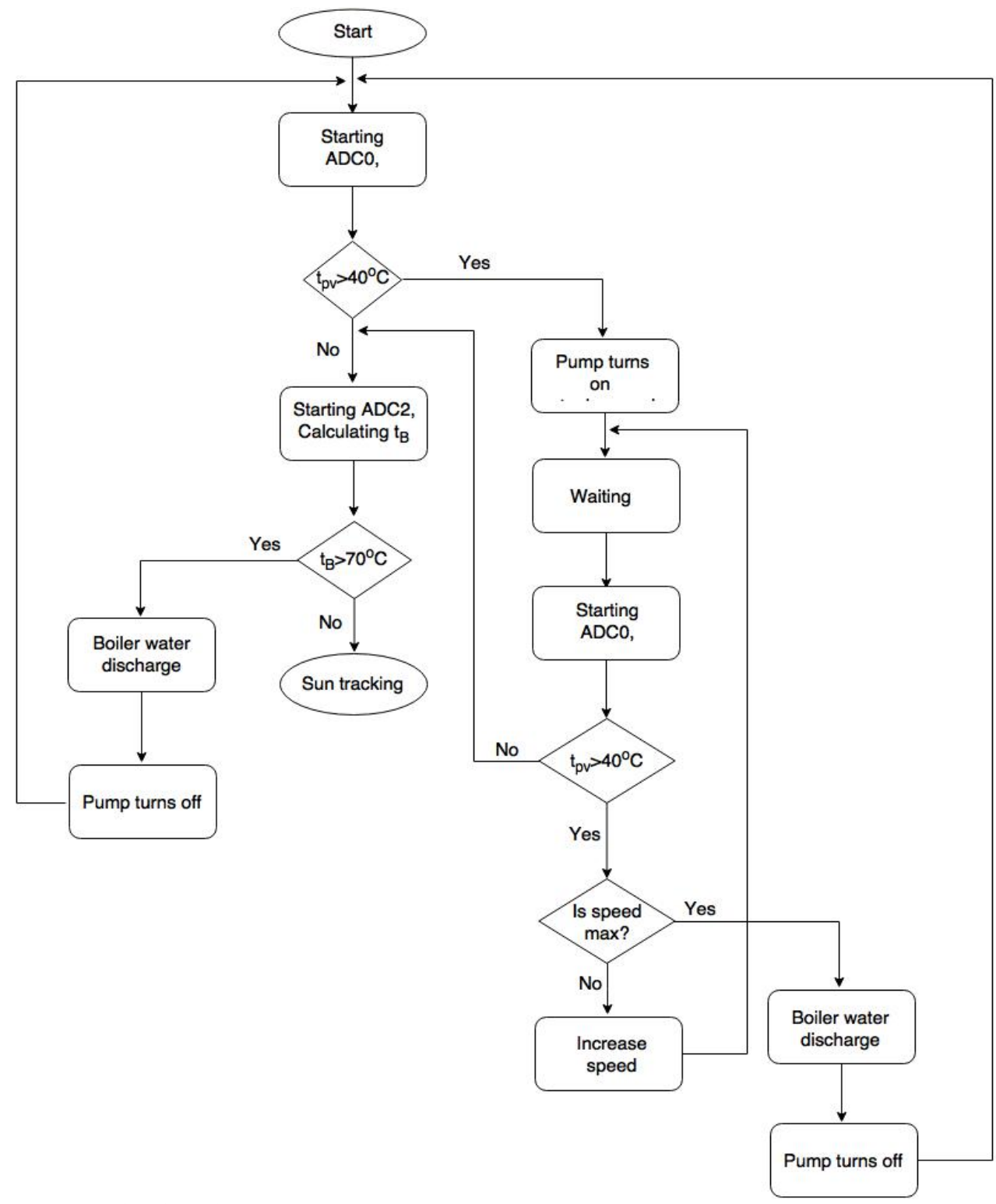

Figure 3. The algorithm of cooling management.

At the beginning the temperature values from the ADC are read from the sensor. Based on these values the temperature of the PV is calculated. Depending on the temperature of the PV the operating mode of the pump is selected. If the temperature of the PV is less than $40^{\circ} \mathrm{C}$, the PV will not be cooled, because it is not necessary. With increasing temperature the speed of circulation of the liquid also increases, which automatically leads to a more intensive heat conduction and, thus, cooling of the system. Once the system temperature reached the threshold of $70^{\circ} \mathrm{C}$ and the speed of circulation its maximum value, water is let out of the boiler. This process is necessary to exchanging hot water cold one, which in turn increases the intensity of the temperature exchange. 


\section{The first experiment}

As can be seen from the field experiment (Figure 4), the heating of the PV surface is a strong (about $85^{\circ} \mathrm{C}$ ). When the cooling is turned on there is a significant cooling (about 30 $\left.{ }^{\circ} \mathrm{C}\right)$.

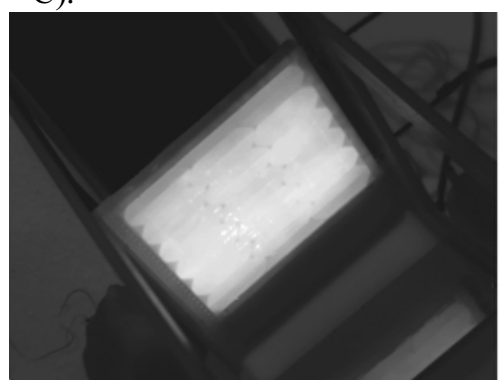

a

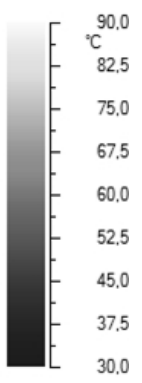

30,0

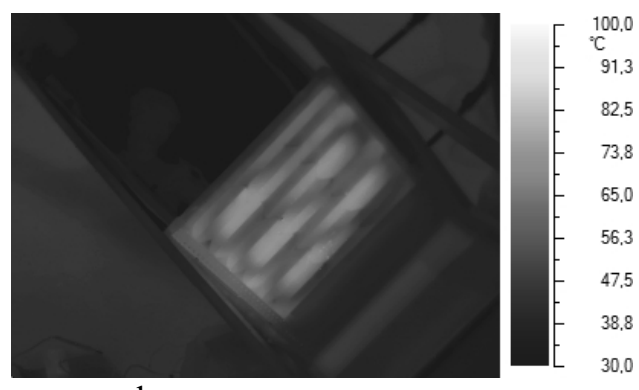

b

Figure 4. The results of the field test: a-without cooling; b-with cooling.

To increase the cooling uniformity in the design can be changed or to add absorber, as well to augment the number of turns of the coil.

According to the results of the experiment can be said that the cooling system is functioning well.

\section{Summary}

The main target group, which this station will be interesting for, are residents of remote and elusive places, tourist accommodations, which are located far away from current paths, foresters, workers of natural reserves, as well as vacationers and gardeners.

It is presented a description of a new hybrid solar system, which uses concentrated sunlight and orientates itself towards the sun. These additional features increase the gain of electrical energy and make it possible to obtain thermal energy. The stored thermal energy may be used in everyday life.

The earlier explained algorithm controls the hybrid solar system. It implements the cooling of the PV and the orientation of the station towards the sun. Particular attention is to pay to the part of the algorithm, which controls the cooling of the PV.

The functioning of an experimental station was proved by an experiment.

\section{Acknowledgment}

The following research has been financially supported by Ministry of Education and Science of the Russian Federation, Agreement № 14.577.21.0018 (RFMEFI57714X0018).

\section{References}

[1] C.-Y. Lee, P.-C. Chou, C.-M. Chiang, C.-F. Lin, Sensors 9, 3875 (2009) doi:10.3390/s90503875

[2] Y.-K. Wua and S.-M. Chang, MATEC Web of Conferences 55, 06001 (2016) doi: 10.1051/matecconf/20165506001

[3] H.A. Zondag, Renew Sustain Energy Rev. 12, $891 \quad$ (2008). doi:10.1016/j.rser.2005.12.012

[4] BeagleBone Black, description URL: http://beagleboard.org/black 
[5] A. Yurchenko, A. Kozloff, A. Okhorzina, M. Kitaeva, A. Purgin, Polzunovsky vestnik (2), 79 (2014) 